Article

\title{
Three-Dimensional Glass Monolithic Micro-Flexure Fabricated by Femtosecond Laser Exposure and Chemical Etching
}

\section{Viktor Tielen and Yves Bellouard *}

Mechanical Engineering Department, Eindhoven University of Technology, 5600 MB Eindhoven, The Netherlands

* Author to whom correspondence should be addressed; E-Mail: yves@bellouard.eu;

Tel.: +31-40-247-3715.

Received: 18 August 2014; in revised form: 4 September 2014 / Accepted: 4 September 2014 /

Published: 11 September 2014

\begin{abstract}
Flexures are components of micro-mechanisms efficiently replacing classical multi-part joints found at the macroscale. So far, flexures have been limited to two-dimensional planar designs due to the lack of a suitable three-dimensional micromanufacturing process. Here we demonstrate and characterize a high-strength transparent monolithic three-dimensional flexural component fabricated out of fused silica using non-ablative femtosecond laser processing combined with chemical etching. As an illustration of the potential use of this flexure, we propose a design of a Hoecken linkage entirely made with three-dimensional cross-spring pivot hinges.
\end{abstract}

Keywords: flexure; femtosecond laser; three-dimensional micromachining; cross-spring pivot; optomechanics; Hoecken (Hoekens) linkage

\section{Introduction}

Flexures are essential mechanical elements commonly used in precision and micro-engineering to precisely guide the motion between mechanical bodies. They consist of combinations of slender, continuous bodies that deform elastically upon the application of a force. Flexures [1-3] have been used for decades in precision instrumentation and mechanisms [4-10]. At the microscale, flexures are used in replacement of traditional, multi-part mechanical joints that cannot be easily miniaturized and that are sensitive to friction, wear, and inaccuracies resulting from assembly errors. 
The design of flexures at the microscale is essentially constrained by the availability of a material and of a suitable machining process. Commonly used fabrication methods have limited flexure design to two-dimensional components.

Here, we demonstrate the fabrication of a three-dimensional flexure - a cross-spring pivot — made out of fused silica glass using femtosecond laser micromachining combined with chemical etching.

This machining process relies on the non-ablative exposure of fused silica to create three-dimensional patterns that are then etched away by immersing the laser-exposed substrate in a chemical solution [11] (typically $\mathrm{HF}$ or $\mathrm{KOH}[12]$ ). This process not only offers the ability to manufacture high-aspect ratio three-dimensional microstructures, but also to integrate structural elements with other functionalities such as optical waveguides [13,14]. This unique capability has paved the way for monolithic optomechanical devices [15] in which flexures are combined with optical waveguides to achieve embedded sensing functions. The machining of three-dimensional structures in a dielectric and transparent substrate such as fused silica offers additional benefits such as the possibility to implement separated conductive tracks and to be optically transparent. As an illustration, a monolithic optically transparent capacitive glass actuator was recently demonstrated [16]. As shown for femtosecond micromachined Foturan glass substrates [17,18], electrodes-less metal plating [19] may also provide an alternative solution for integrating electrical functionalities. Finally, recent advances in beam shaping methods (like for instance the use of holographic methods [20]) will further expand femtosecond laser processing capabilities.

Although counterintuitive at first, fused silica (the glass used in this study), has a very high mechanical strength if its surface is free of defects. In particular, we demonstrated that flexures with an ultimate tensile strength in excess of $2 \mathrm{GPa}$ can be manufactured with femtosecond laser processing [21]. These high stress levels are well above those (usually below $500 \mathrm{MPa}$ ) typically found in commonly used flexure construction materials such as spring steel or aluminum used at the macro-scale.

Finally, in this paper, we illustrate the design of a complete Hoecken linkage [22-24] utilizing three-dimensional cross-pivots as an example of complex monolithic mechanical devices.

\section{The Cross-Pivot as an Elemental Flexure}

\subsection{Working Principle and Relevance}

In flexure-based mechanisms, a pivot function is replaced with cantilevers, notch hinges or cross-spring pivots. An ideal pivot flexure should have a fixed center of rotation, a small stiffness along the pivoting degree of freedom and an "infinitely" high stiffness along the other unwanted degrees of freedom. The later property is referred to the flexure stiffness contrast.

The cantilever is a hinge with a large range of motion because of its ability to spread the stress across its entire geometry. As it will be emphasized later, it suffers from the drift of the point of rotation and from a weak stiffness contrast, making it a three-degree-of-freedom flexure as opposed to a single degree of freedom as prescribed for a pivot function. The classical notch hinge has a well-defined point of rotation, which helps in mimicking conventional, friction-based joints, but is subject to detrimental high stress concentrations. Like for the cantilever, it also suffers from a poor stiffness contrast.

The cross pivot hinge, shown in Figure 1, combines the appealing properties of a cantilever and a notch hinge into one. Its crossed structure limits the drift of the point of rotation while keeping large range motion, and the lack of notches prevents stress concentrations. The main limitation of the 
cross-pivot is its inherent geometrical complexity and its non-trivial, intrinsically three-dimensional shape. In particular, at the micro-scale, such a flexure cannot be fabricated by conventional means such as commonly used lithographic processes like so-called Deep Reactive Ion Etching (DRIE).

Figure 1. Working principle of a cross-spring pivot. (a) Geometrical definition: the cross-spring pivot - in its simplest form — consists of two leaf-springs defining a cross that connects two rigid bodies. (b) Three-dimensional rendering of the deformed shape of a classical cross-pivot upon the application of a pure moment (from an actual finite element simulation). The light grey shadow shows the structure at rest. The cross-spring mimics a pivot joint function. (c) Side view schematic. The rotation axis of the hinge is found at the intersection between the two leaf-springs. While deforming, the position of the center of rotation is subject to a parasitic displacement.
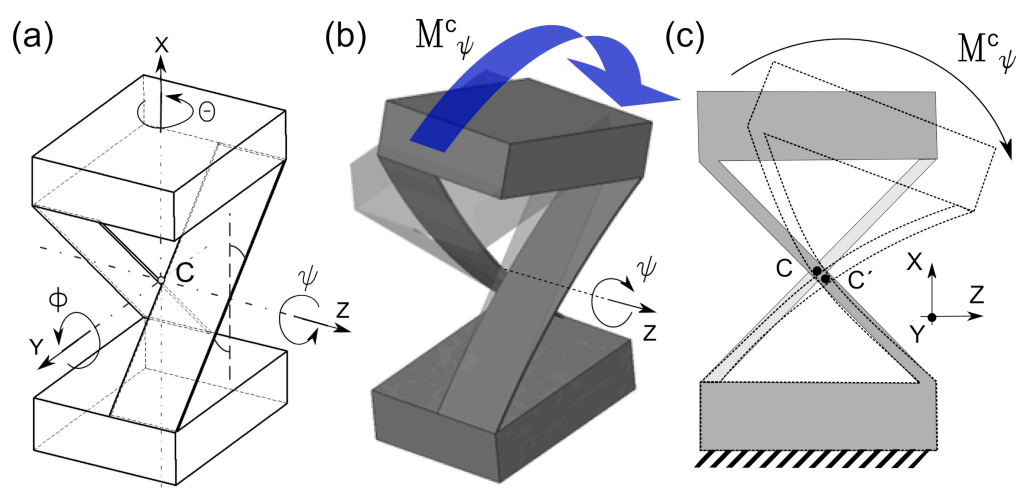

\subsection{Stiffness Modeling of the Classical Cross-Spring Pivot}

A convenient way to rapidly derive the stiffness properties of complex assembly of flexural elements is to use a method derived from robotics and screw theory [25-27]. The stiffness matrix for a flexure that connects two rigid bodies (labelled 0 and 1) and expressed at an arbitrarily chosen point $\mathrm{P}$, in its generalized form can be written as:

$$
\left(\hat{\mathrm{f}}_{1 / 0}^{\mathrm{P}}\right)=\mathrm{K}^{\mathrm{P}}\left(\hat{\mathrm{r}}_{1 / 0}^{\mathrm{P}}\right)^{*} \Rightarrow\left[\begin{array}{c}
\mathrm{F}_{1 / 0} \\
\mathrm{M}_{1 / 0}^{\mathrm{P}}
\end{array}\right]=\left[\begin{array}{cc}
\mathrm{K}_{\mathrm{F}, \mathrm{u}}^{\mathrm{P}} & \mathrm{K}_{\mathrm{F}, \theta}^{\mathrm{P}} \\
\mathrm{K}_{\mathrm{M}, \mathrm{u}}^{\mathrm{P}} & \mathrm{K}_{\mathrm{M}, \theta}^{\mathrm{P}}
\end{array}\right]\left[\begin{array}{c}
\mathrm{u}_{1 / 0}^{\mathrm{P}} \\
\theta_{1 / 0}
\end{array}\right]
$$

Where the left term $\mathbf{f}$ represents the wrench (a six-coordinates vector containing force and moment) and the right term the $6 \times 6$ generalized stiffness matrix $\mathbf{K}$ times a displacement spatial vector $\mathbf{r}$ that contains a linear displacement and an angular component. In the case of the cross-spring pivot, the methodology (described in great detail in [27]) for deriving the generalized stiffness, consists of, first, expressing the stiffness matrix of the beams [28] in a local coordinate frame and center of inertia, and second, to use coordinate transformations (here a rotation of $\pi / 4$ and a translation of half the beam width) to express the two springs' stiffnesses at the same point and in the same coordinate frame.

The resulting stiffness matrix for the cross-spring pivot is simply the sum of the two elementary spring matrices, since both are connected in parallel to the same bodies. The end result is shown below. In these formulas, the flexure component of lowest stiffness that defines its preferred degree-of-freedom is highlighted in a box. 


$$
\left[\begin{array}{c}
F_{x} \\
F_{y} \\
F_{z} \\
M_{\theta}^{C} \\
M_{\phi}^{C} \\
M_{\psi}^{C}
\end{array}\right]=\left[\begin{array}{cccccc}
k_{F_{x}, x}^{C} & 0 & 0 & k_{F_{x}, \theta}^{C} & 0 & 0 \\
0 & k_{F_{y}, y}^{C} & 0 & 0 & k_{F_{y}, \phi}^{C} & 0 \\
0 & 0 & k_{F_{z}, z}^{C} & 0 & 0 & 0 \\
k_{M_{\theta}, x}^{C} & 0 & 0 & k_{M_{\theta}, \theta}^{C} & 0 & 0 \\
0 & k_{M_{\phi}, y}^{C} & 0 & 0 & k_{M_{\phi}, \phi}^{C} & 0 \\
0 & 0 & 0 & 0 & 0 & k_{M_{\psi}, \psi}^{C}
\end{array}\right]\left[\begin{array}{c}
\Delta_{x} \\
\Delta_{y} \\
\Delta_{z} \\
\Delta_{\theta} \\
\Delta_{\phi} \\
\Delta_{\psi}
\end{array}\right]
$$

With the diagonal terms expressed as follow:

$$
\left\{\begin{array} { c } 
{ k _ { F _ { x } , x } ^ { C } = \frac { E w t } { L } + \frac { E w t ^ { 3 } } { L ^ { 3 } } } \\
{ k _ { F _ { y } , y } ^ { C } = \frac { E w t } { L } + \frac { E w t ^ { 3 } } { L ^ { 3 } } }
\end{array} \text { and } \left\{\begin{array}{c}
k_{M_{\theta}, \theta}^{C}=\frac{E w^{3} t}{12 L}+\frac{E w^{3} t^{3}}{2 L^{3}}+\frac{E w t^{3}}{6 L(v+1)} \\
k_{F_{z}, z}^{C}=\frac{2 E w^{3} t}{L^{3}}
\end{array}\right.\right.
$$

The non-diagonal terms are:

$$
k_{F_{x}, \theta}^{C}=k_{M_{\theta}, x}^{C}=\frac{\sqrt{2} E w^{2} t^{3}}{2 L^{3}} \quad \text { and } \quad k_{F_{y}, \phi}^{C}=k_{M_{\phi}, y}^{C}=\frac{\sqrt{2} E w^{2} t}{2 L}
$$

In these formulas, $E, w, t$ and $L$ are the Young modulus of the spring material, the width, the thickness, and length of the individual springs, respectively. $v$ is the Poisson coefficient of the spring material. The axis notations and the location of point $\mathrm{C}$ are shown in Figure 1a.

Non-diagonal terms in the stiffness matrix can be undesired as it implies non-pure transformation from a given axis of application of force into an actual displacement along another axis. For instance, a force applied along the $y$-axis will result in a displacement along the $y$-axis as well as an out-of-plane deformation by rotation along the same axis. In the ideal case, only a pure rotation would be applied around the $z$-axis to activate the pivot-function. In practice, pivot flexures are integrated into more complex mechanisms and the moment causing the pivot rotation is not just along a single axis but rather a combination of force and moment (wrench). In order to achieve high precision in the design of a planar mechanism that uses cross-spring pivots, it is useful to cancel non-diagonal terms that can be activated through in-plane forces and that may cause unwanted, out-of-plane parasitic displacements. Some of the non-diagonal terms can be zeroed through optimization of the flexure design as it will be shown in the next paragraph.

\subsection{Design Optimization}

By adding a third beam, two of the non-diagonal terms are efficiently suppressed. The optimized design is shown in Figure 2. The shape is further optimized using finite element modeling for reducing stress concentration in a monolithic design (Figure 2c). 
Figure 2. (a) 3D rendering of the optimized design for the monolithic cross-spring pivot hinge, showing the addition of the third beam and the removal of sharp edges in geometry to prevent high stress concentrations. Point $\mathrm{C}$ refers to the origin of the coordinate frame. (b) Finite element simulation of the designed hinge subjected to a moment applied around the $\mathrm{z}$ axis. The model is meshed using tetrahedral elements of $3 \mu \mathrm{m}$ at the thinnest part of the crossed beams. (c) Geometrical parameters for a single beam.

(a)

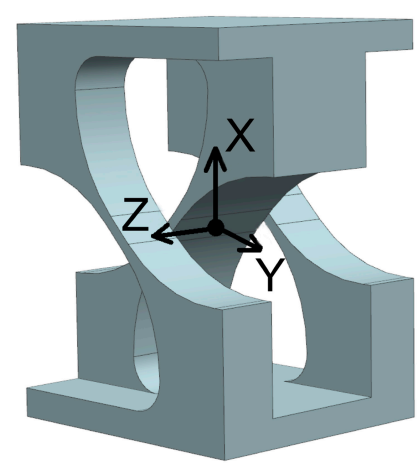

(b)

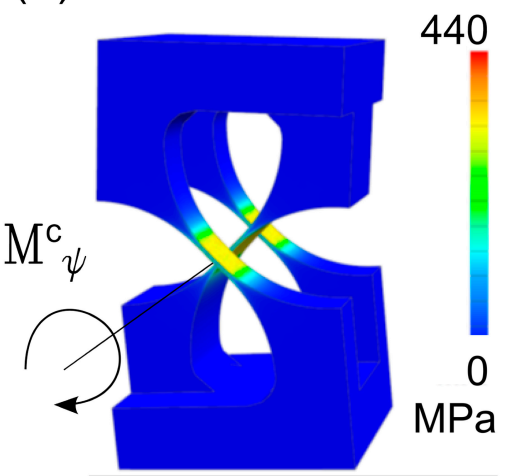

(c)

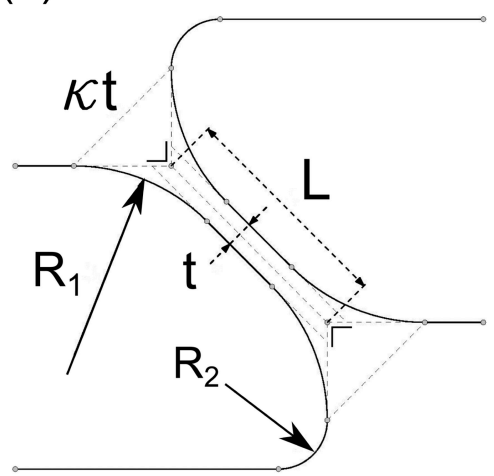

Compared to the original matrix in (2), in matrix (5) shown below, the off-diagonal terms are replaced by two different ones that relate to an out-of-plane coupling (moment applied around $\theta$ and $\phi$ ) that cannot be activated by a force applied in the geometrical plane of operation for the hinge. (Note that we keep the same axis notations than in the previous case. This time point $\mathrm{C}$ is located in the middle of the middle bar, i.e., the origin of the coordinate frame in Figure 2a.)

$$
\left[\begin{array}{c}
F_{x} \\
F_{y} \\
F_{z} \\
M_{\theta}^{C} \\
M_{\phi}^{C} \\
M_{\psi}^{C}
\end{array}\right]=\left[\begin{array}{cccccc}
k_{F_{x}, x}^{C} & 0 & 0 & 0 & 0 & 0 \\
0 & k_{F_{y}, y}^{C} & 0 & 0 & 0 & 0 \\
0 & 0 & k_{F_{z}, z}^{C} & 0 & 0 & 0 \\
0 & 0 & 0 & k_{M_{\theta}, \theta}^{C} & k_{M_{\theta}, \phi}^{C} & 0 \\
0 & 0 & 0 & k_{M_{\phi}, \theta}^{C} & k_{M_{\phi}, \phi}^{C} & 0 \\
0 & 0 & 0 & 0 & 0 & k_{M_{\psi}, \psi}^{C}
\end{array}\right]\left[\begin{array}{c}
\Delta_{x} \\
\Delta_{y} \\
\Delta_{z} \\
\Delta_{\theta} \\
\Delta_{\phi} \\
\Delta_{\psi}
\end{array}\right]
$$

With the stiffness terms written as (the natural degree of freedom stiffness is emphasized):

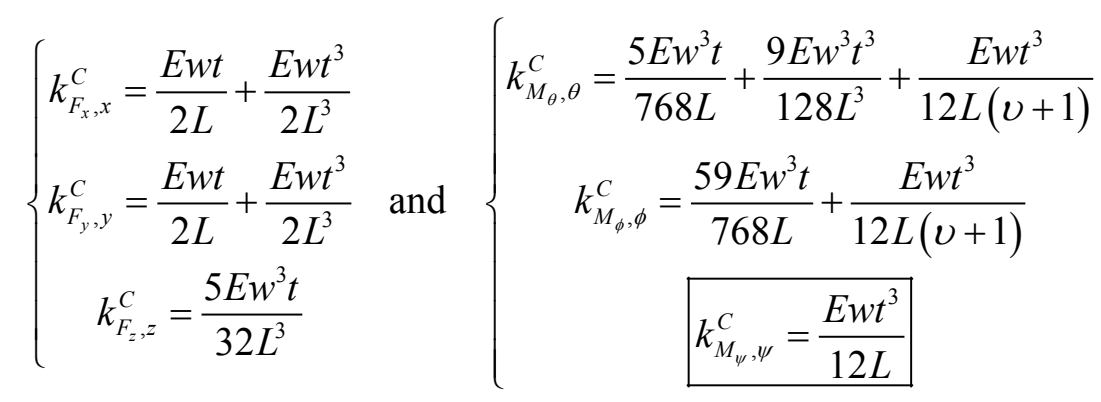

In these equations, $w$ is the thickness of the central beam. The two thinner, outer beams have a width of $w / 2$. The stiffness of the remaining non-diagonal term is: 


$$
k_{M_{\phi}, \theta}^{C}=k_{M_{\theta}, \phi}^{C}=-\frac{E w^{3} t}{256 L}
$$

And the parametric equation that defines the curvature of the anchoring points for the beams (see Figure $2 \mathrm{c}$ for the meaning of the various parameters) that prevents stress concentration is expressed as:

$$
R_{1}=\frac{t(\kappa-1)}{2\left[1-(\sqrt{2})^{-1}\right]} \quad \text { and } \quad R_{2}=\frac{L}{6}
$$

To qualitatively compare the performances of cross-spring pivots with other flexure pivot designs, we examine the stiffness contrast of the optimized design. By stiffness contrast, we mean the ratio between angular and linear stiffness along each axis. To do so, we consider the weakest stiffness and evaluate the ratio of this stiffness with the other stiffer axis. An ideal pivot should have the highest possible contrast between the stiffness of its natural degree of freedom and that of the other axis. To make the comparison effective and to have it only dependent on one parameter, we assume the same scaling factor between the length of the beam versus its widths and the width versus its thickness. This translates into the following expression, where $\mathrm{s}$ is a scaling factor:

$$
L=s \cdot w=s^{2} \cdot t
$$

A typical order of magnitude for s to satisfy well beam-theory conditions is $s \geq 10$.

Introducing the expression (9) above in the stiffness relations for the optimized cross-pivot, yields:

$$
\begin{aligned}
& \left\{\begin{array}{l}
k_{F_{x}, x}^{C}=k_{F_{y}, y}^{C}=\frac{E t}{s}+\frac{E t}{s^{5}} \approx \frac{E t}{s} \approx \frac{4}{5} s^{2} k_{F_{z}, z}^{C} \\
k_{F_{z}, z}^{C}=\frac{5 E t}{4 s^{3}} \simeq \frac{5}{4 s^{2}} k_{F_{x}, x}^{C}
\end{array}\right. \\
& \left\{\begin{array}{l}
k_{M_{\theta}, \theta}^{C}=\frac{5 E s t^{3}}{96}+\frac{9 E t^{3}}{16 s^{3}}+\frac{E t^{3}}{6 s(v+1)} \approx \frac{5 E s t^{3}}{96}+\frac{E t^{3}}{6 s(v+1)} \approx \frac{5}{16} s^{2} k_{M_{\psi}, \psi}^{C} \\
k_{M_{\phi}, \phi}^{C}=\frac{59 E s t^{3}}{96}+\frac{E t^{3}}{6 s(v+1)} \approx \frac{59}{16} s^{2} k_{M_{\psi}, \psi}^{C} \\
k_{M_{\psi}, \psi}^{C}=\frac{E t^{3}}{6 s}
\end{array}\right.
\end{aligned}
$$

A pure moment is difficult to apply in practice, especially in a complex design involving more than one pivot flexure. Instead, a pseudo-moment is applied, i.e., a force off the axis passing through the pivot center of rotation, that eventually induces the hinge rotation. Since in flexures, stiffnesses are finite, the force applied to generate the pivoting moment also induces a displacement along its axis that slightly modifies the loading case, and may create a parasitic displacement. It is therefore useful to examine which displacement will prevail in such situation (between angular- the desired pivot rotation and linear - the parasitic motion). To compare angular stiffness (in N.m/rad) and linear stiffness $(\mathrm{N} / \mathrm{m})$, we compare a force applied along the rotation axis at a characteristic distance $L$ (equivalent to the characteristic length of the longest element of the beam) from the pivot axis.

For the cross-spring pivot, we obtain (using the axis notations from Figure 2): 


$$
\left.\begin{array}{l}
M_{\psi}^{O}=F_{y} \cdot L=\frac{E t^{3}}{6 s} \Delta_{\psi} \Rightarrow \Delta_{\psi}=\frac{6 s}{E t^{3}} \cdot L F_{y}=\frac{6 s^{3}}{E t^{2}} \cdot F_{y} \\
\Rightarrow \Delta_{y}^{e q}=L \cdot \Delta_{\psi}=\frac{6 s^{5}}{E t} \cdot F_{y} \\
F_{y}=k_{F_{y}, y}^{C} \Delta_{y}=\left(\frac{E w t}{L}+\frac{E w t^{3}}{L^{3}}\right) \Delta_{y} \Rightarrow \Delta_{y} \simeq \frac{s}{E t} F_{y}
\end{array}\right\}
$$

This result can be interpreted as follows: a force $F$ applied at a distance $L$ from the center of rotation and along the $y$ axis will induce a pivoting moment along the $z$ axis, in addition to the force $F$ along the $y$ axis. The force along the $y$ axis induces a natural parasitic displacement along $y$ of $\Delta_{y}$. This parasitic displacement is compared to the amplitude of displacement, called $\Delta_{y}^{e q}$, along the same axis but caused by the rotation of the hinge. In the ideal situation of a perfect pivot, the ratio between $\Delta_{y}^{e q}$ and $\Delta_{y}$ should be infinite. Using the same methodology, we compare the performances of the optimized cross-spring pivot in terms of stiffness contrast and off-axis behavior with those of a classical cantilever with similar characteristic dimensions (i.e., length $L$, width $w$ and thickness $t$ ). The results are shown in the Table 1.

Table 1. Stiffness contrast for the cross-spring pivot, compared with a cantilever, one of the most used flexure as pivot.

\begin{tabular}{cccccccc}
\hline Stiffness component & $k_{F_{x}, x}^{C}$ & $k_{F_{y}, y}^{C}$ & $k_{F_{z}, z}^{C}$ & $k_{M_{\theta}, \theta}^{C}$ & $k_{M_{\phi}, \phi}^{C}$ & $k_{M_{\psi}, \psi}^{C}$ & $\frac{\Delta_{y}^{e q}}{\Delta_{y}}$ \\
\hline Cross-spring pivot & $\frac{4}{5} s^{2}$ & $\frac{4}{5} s^{2}$ & 1 & $\frac{5}{16} s^{2}$ & $\frac{59}{16} s^{2}$ & 1 & $6 s^{4}$ \\
Cantilever & $\mathrm{s}^{4}$ & 1 & $\mathrm{~s}^{2}$ & $\mathrm{~s}^{2}$ & {$\left[\frac{2}{(1+\mu)}\right]$} & 1 & 12 \\
\hline
\end{tabular}

In this table, the ratios between the lowest stiffness (either angular or linear) and the other stiffness are compared. This table reveals that the cross-spring pivot shows superior stiffness contrast for angular displacement. Indeed, in the case of a cantilever, the hinge can not only pivot along the $z$-axis (as desired) but also remains weak in torsion. Furthermore, if an off-axis force is applied to induce the pivoting of a cantilever, it will induce an undesired displacement along the $y$-axis of similar amplitude. A cantilever taken alone can therefore be seen as a three-degree of freedom flexure: It has rather low-stiffness as pivot along the $z$-axis but also in torsion (around the $y$-axis) as well as for a linear displacement along the $y$-axis. In contrast, the cross-spring pivot has globally a high stiffness contrast and is closer to a pure pivot. It can be considered-in the flexure approximation of a degree-of-freedom - as a one-degree-of-freedom pivot. Note that the comparison of Table 1 can be extended to the notch hinge and would yield similar results. Indeed, the notch hinge has similar stiffness contrast behavior to that of a cantilever beam.

The performance of a flexure can also be assessed in terms of maximum angular deflection versus flexure dimensions. The maximum angle for the cross-spring pivot (for both designs, optimized or not) is given by: 


$$
\psi_{\max }=\frac{2 \sigma_{L} L}{E t}
$$

In which $\sigma_{L}$ is the elastic limit of the material considered, $L$ the longest length of the beam, $E$ the Young's modulus and $t$ the beam thickness, respectively.

To summarize, in Table 2, we propose a qualitative comparison between the three types of hinge commonly used as pivots. As evaluation criteria, we compare the maximum angular deflection as well as other key performance factors such as drift of the center of rotation and stress concentration.

Table 2. Performance comparison between commonly used elemental flexures and a cross-spring pivot.

\begin{tabular}{|c|c|c|c|c|}
\hline \multirow{2}{*}{ Property } & \multicolumn{4}{|c|}{ Hinge type } \\
\hline & Cantilever * & Notch hinge & Cartwheel [1] & Cross-spring pivot ${ }^{* * *}$ \\
\hline $\begin{array}{l}\text { Drift of the center of rotation } \\
\text { (assuming a perfect loading } \\
\text { case, i.e., a pure moment } \\
\text { applied on the hinge) }\end{array}$ & $\delta=\zeta \mathrm{L} \theta^{2}$ & $\sim 0{ }^{* *}$ & $\begin{array}{l}\delta \simeq \frac{\sqrt{2}}{30} L \theta^{2} \\
\left(\simeq 0.047 L \theta^{2}\right)\end{array}$ & $\delta \simeq \lambda \mathrm{L} \theta^{2}$ \\
\hline Maximum angular deflection & $\frac{\mathrm{L}}{\mathrm{t}}\left(\frac{\sigma_{\max }}{\mathrm{E}}\right)$ & $\simeq \frac{3 \pi}{4} \sqrt{\frac{\mathrm{L}}{\mathrm{t}}}\left(\frac{\sigma_{\max }}{\mathrm{E}}\right.$ & $\frac{\mathrm{L}}{2 \mathrm{t}}\left(\frac{\sigma_{\max }}{\mathrm{E}}\right)$ & $2 \frac{\mathrm{L}}{\mathrm{t}}\left(\frac{\sigma_{\max }}{\mathrm{E}}\right)$ \\
\hline Stress concentration & Low & High & Medium & Low \\
\hline Complexity/manufacturability & Low & Medium & Medium & High (3D) \\
\hline
\end{tabular}

Notes: ${ }^{*}$ For a cantilever, the center of rotation is defined at one third of the cantilever length. The constant $\zeta$ is fitted to be approximately 0.0158 . ${ }^{* *}$ The drift is order of magnitude smaller than the thinnest part of the hinge itself and therefore negligible. ${ }^{* * *}$ The constant $\lambda$ is 0.118 [6], 0.112 [5], 0.125 [7]. A typical order of magnitude of the rotation center displacement is about $4 \%$ of the beam length for a deflection of $45^{\circ}$.

Cantilever, cross-spring and cartwheel pivots present a drift of the center of rotation while operated. The drift is the highest for the cross-spring pivot but remains within a few percent of the beam length. In both cases, stress concentrations are avoided. The cross spring pivot, for similar characteristic dimensions, has two times more angular deflection than the cantilever beam and four times more than a cartwheel hinge [1,29].

Considering this quantitative comparison between the various flexures used as pivots, the cross-spring pivot has a significant number of key advantages, including a high-stiffness contrast that defines a single-degree of freedom and the absence of stress-concentration. The main challenge is its inherent three-dimensional structure, which is difficult to manufacture. While it can be relatively easily implemented at the macro-scale by assembling leaf-springs on a support structure, it has to date never been made in miniaturized versions suitable for microsystems applications.

In the next section, we demonstrate the fabrication of an optimized cross-spring pivot using femtosecond laser processing.

\section{Experimental Results}

\subsection{Device Fabrication}

A scanning electron microscope (SEM) and an optical microscope image of a cross-spring pivot fabricated with femtosecond laser and chemical etching are shown in Figure 3. The device is fabricated 
using a two-step process described in great detail elsewhere [30]. The first step is to expose a 500- $\mu \mathrm{m}$ thick silica substrate (synthetic silica with high $\mathrm{OH}$ content) to low-energy pulses, below the ablation threshold. During this step, the material structure is locally modified, resulting in an accelerated HF etching rate in the laser exposed regions.

The exposure step was done with 380 fs-long pulses emitted at $1030 \mathrm{~nm}$ from a diode pumped Ytterbium-KGW based femtosecond oscillator (t-Pulse 500 from Amplitude Systèmes, Bordeaux, France). The oscillator emits pulses at a frequency of 9.4 MHz. An acousto-optic modulator (AOM) was used to reduce the repetition rate by chopping out pulses from the main pulse train. For this device, we used a repetition rate of $860 \mathrm{kHz}$. The laser beam was focused using a $20 \times$ objective (OFR-20X-1054, Thorlabs, NJ, USA) with a numerical aperture (NA) of 0.40 . Using three linear positioning stages, the specimen was moved with an accuracy of $1 \mu \mathrm{m}$ under the objective. The writing speeds were 5 and $8 \mathrm{~mm} / \mathrm{s}$ and the pulse energy was $215 \mathrm{~nJ}$.

The second process step is chemical etching. The laser modified-substrate is dipped into a low-concentration HF bath (from $2.5 \%$ to $5 \%$ ) for $18 \mathrm{~h}$. Although the etchant exposure time varied across the thickness, the tapered profile does not exceed $2 \mu \mathrm{m}$ over a thickness of $300 \mu \mathrm{m}$ demonstrating the high-aspect ratio capability of the process.

Figure 3. Scanning Electron Microscope image (left) and microscope image (right) of a cross-pivot hinge as part of a mechanism. The images show the three, crossed beams and the rounded corners at the location where the beams are connected to the main body. Note the high aspect ratio of the micromachining process.
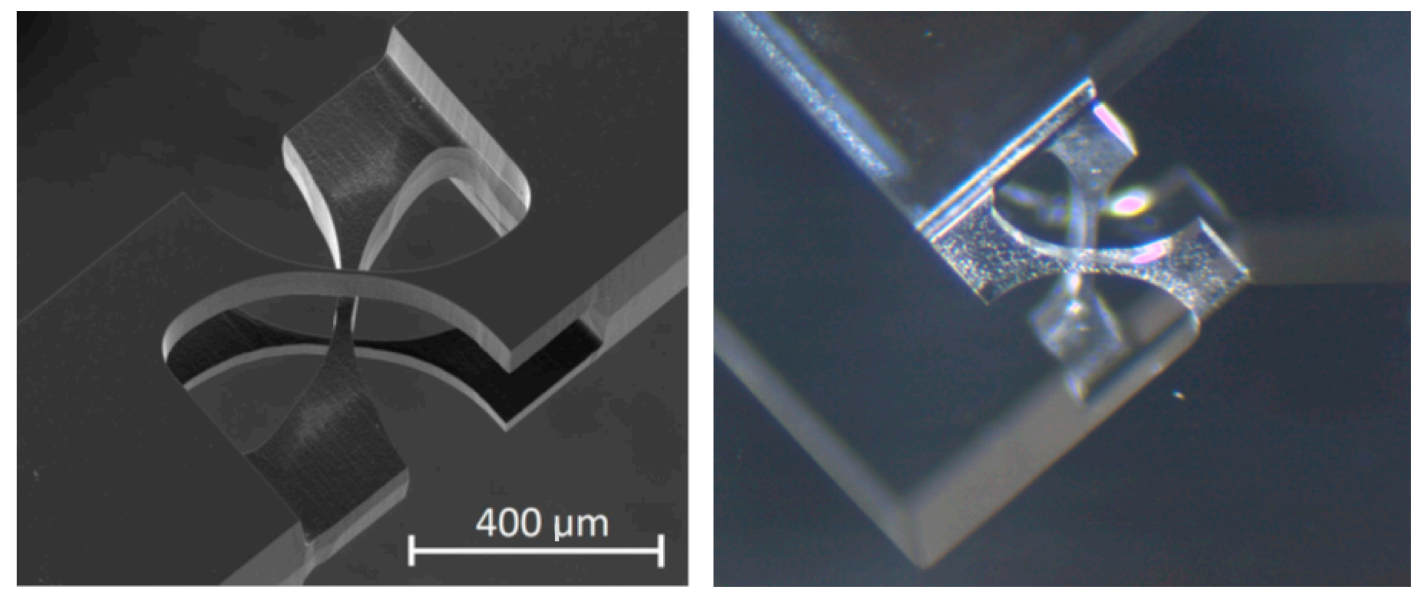

\subsection{Stress-Induced Birefringence: Direct Measurement of Stress State in the Flexure}

Using transparent substrates in micromechanics offers a distinctive advantage: stress can be observed directly through photoelastic effects. Indeed, the presence of stress induces birefringence that can be quantified and monitored by measuring the retardance and when necessary, the orientation of the principal axes of the birefringence ellipse. In [21], we use photoelasticity to demonstrate that femtosecond laser micromachined notch hinges could withstand very high stress levels, typically above $2 \mathrm{GPa}$. While it is known that amorphous silica has a theoretically high elastic limit, it has a low fracture toughness that limits its use as a mechanical component. The main difficulty is to achieve sufficiently high surface quality to prevent the formation of cracks. We showed [21] that the etching 
step in this manufacturing process contributes to extending the maximum stress limit sustainable by femtosecond laser micromachined structure. Here, we use a polarization microscope (LC-Polscope ${ }^{\circledR}$, CRi, Cambridge, MA, USA) to measure the retardance through a thick cross-pivot structure. The results are shown in Figure 4.

Figure 4. Retardance image (left) of a cross-pivot being deformed, coded in grey levels. The average beam thickness is $60 \mu \mathrm{m}$ and the bending angle 13 degrees. The observed retardance results from the stress developing in the hinge. By measuring the grey level on the retardance scale (right) modulated by the number of visible fringes, starting from the center were the stress is minimum, the effective maximum stress, $1.9 \mathrm{GPa}$, present in the flexure is determined using the photoelastic coefficients of fused silica (see [21] for more details).
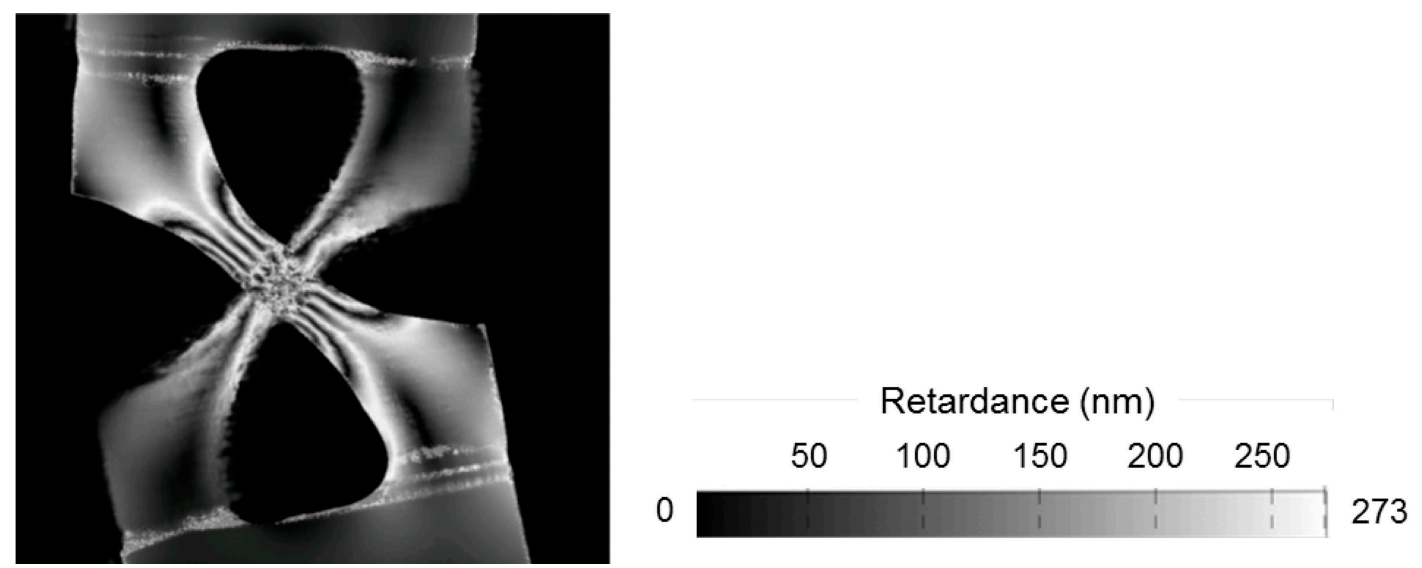

The result in Figure 4 illustrates the stress-state in the material. Since the lever distance-where the force is applied - is several times more than the length of the flexure itself (parameter $L$ in the previous paragraph), and since the angular stiffness around the $z$-axis is much lower than the linear stiffness in the $y$-direction, the loading case can be approximated as a pure bending loading case, which is confirmed by these observations. In this flexure, the hinge thickness is rather high $(60 \mu \mathrm{m})$ leading to high stress level (1.9 GPa in Figure 4) for an angular deformation of 13 degrees from its rest position.

In the following paragraph, we investigate the use of a cross pivot hinge for practical application, such as a linear guidance.

\subsection{Integration of the Cross-Spring Flexure in a Pure Linear Guidance}

A Hoecken guidance (see Figure 5) [22-24] is a quasi-rectilinear guidance made of four pivot joints. It converts a rotational motion into an approximately constant velocity linear motion.

Such rectilinear guidance mechanisms are difficult to design using classical hinges due to the range motion requirements (inducing high-stress and large deflection in the hinge) as well as the joints spatial arrangements causing a non-uniform loading of the pivots. Cross-spring pivots offer an interesting solution since the load is distributed instead of being concentrated. Furthermore, as illustrated in the previous paragraph, for the same maximum stress value, a cross-spring pivot can flex over a larger range and has a higher stiffness contrast, making these stiffness characteristics closer to an ideal one-degree-of-freedom pivot. Figure 6 shows a monolithic glass Hoecken guidance that uses four cross-springs as pivots. 
Figure 5. Working principle of a four-bar Hoecken's linkage. The joints are located in A, $\mathrm{B}, \mathrm{C}$ and $\mathrm{D}$. The characterized lengths of the segment for this example are indicated on the right. A and B are attached to the fixed world. During operation, point $\mathrm{M}$ describes a trajectory characterized with a nearly rectilinear section. This linkage effectively converts a rotation (in A or B) into a quasi-straight motion in $\mathrm{M}$ with constant velocity.

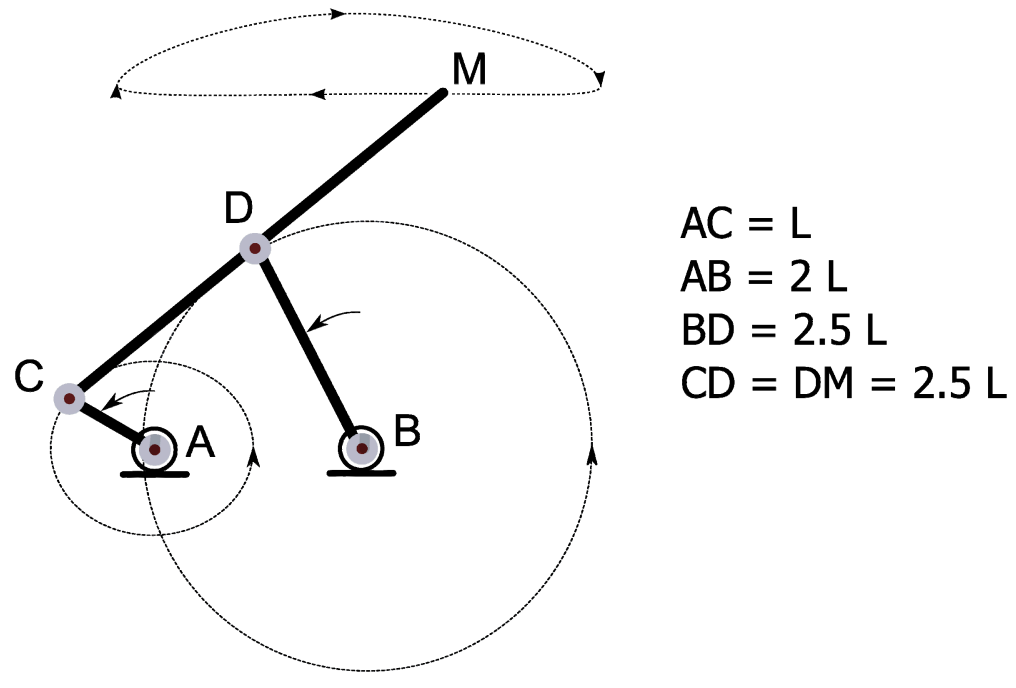

Figure 6. A monolithic glass Hoecken linear guidance (left) and a sequence of merged images to illustrate the function of the mechanism (right). The end point-i.e., where the linear motion is produced-is the cross in the upper part. The mechanism is activated by moving the rectangular window (left side of the images) along a circle. The linearity is within $\pm 0.5 \mu \mathrm{m}$ over a displacement of $600 \mu \mathrm{m}$.
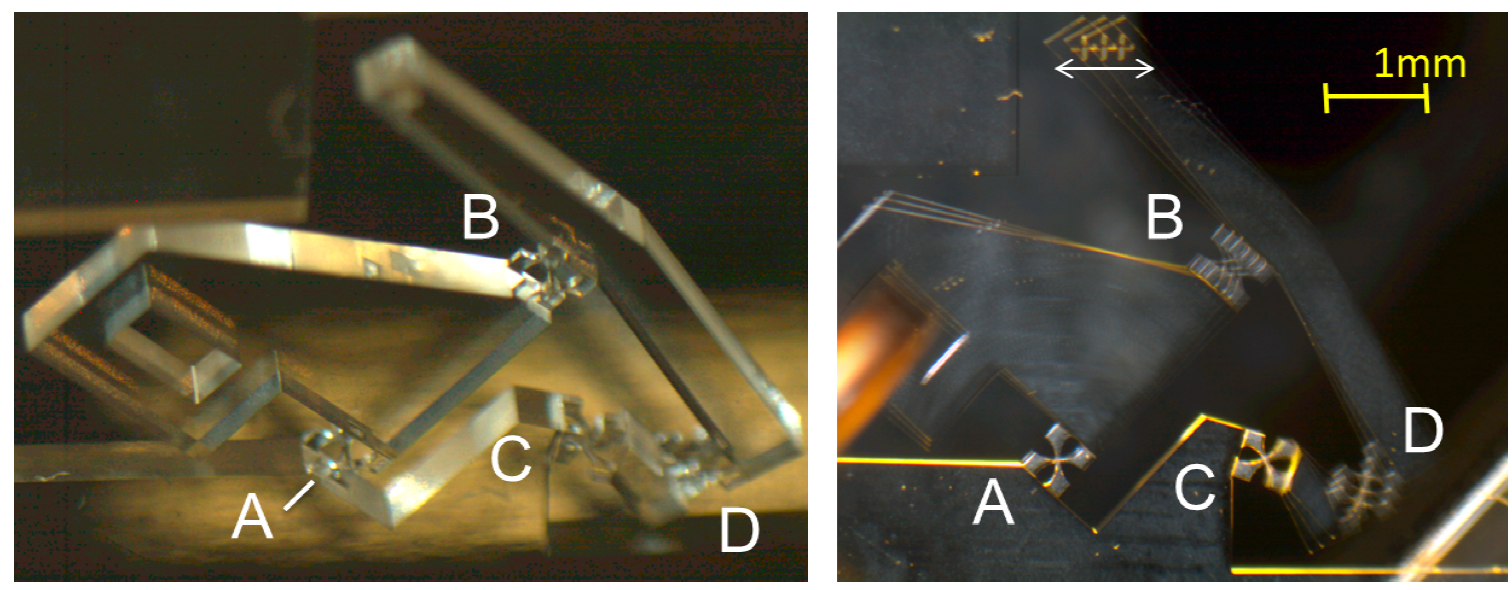

The guidance is entirely manufactured using femtosecond laser micromachining according the process described earlier. The left image shows the complete guidance and the right image (a superposition of images), the deformed structure during operation.

Figure 7 shows the trajectory in the plane of the various key points of the guidance. The dotted lines are the theoretical trajectory for a Hoecken linkage when pivots achieve a complete revolution. The solid lines are the trajectory of the point in the case of the flexure, for which pivots have limited excursions. 
Figure 7. (Left): Trajectory of various key points selected on the Hoecken linear guidance (as shown in the (right) photograph). The dotted lines are the theoretical trajectory for a full rotation of the pivots while the continuous line is the actual range motion of the flexure cross-spring pivots.

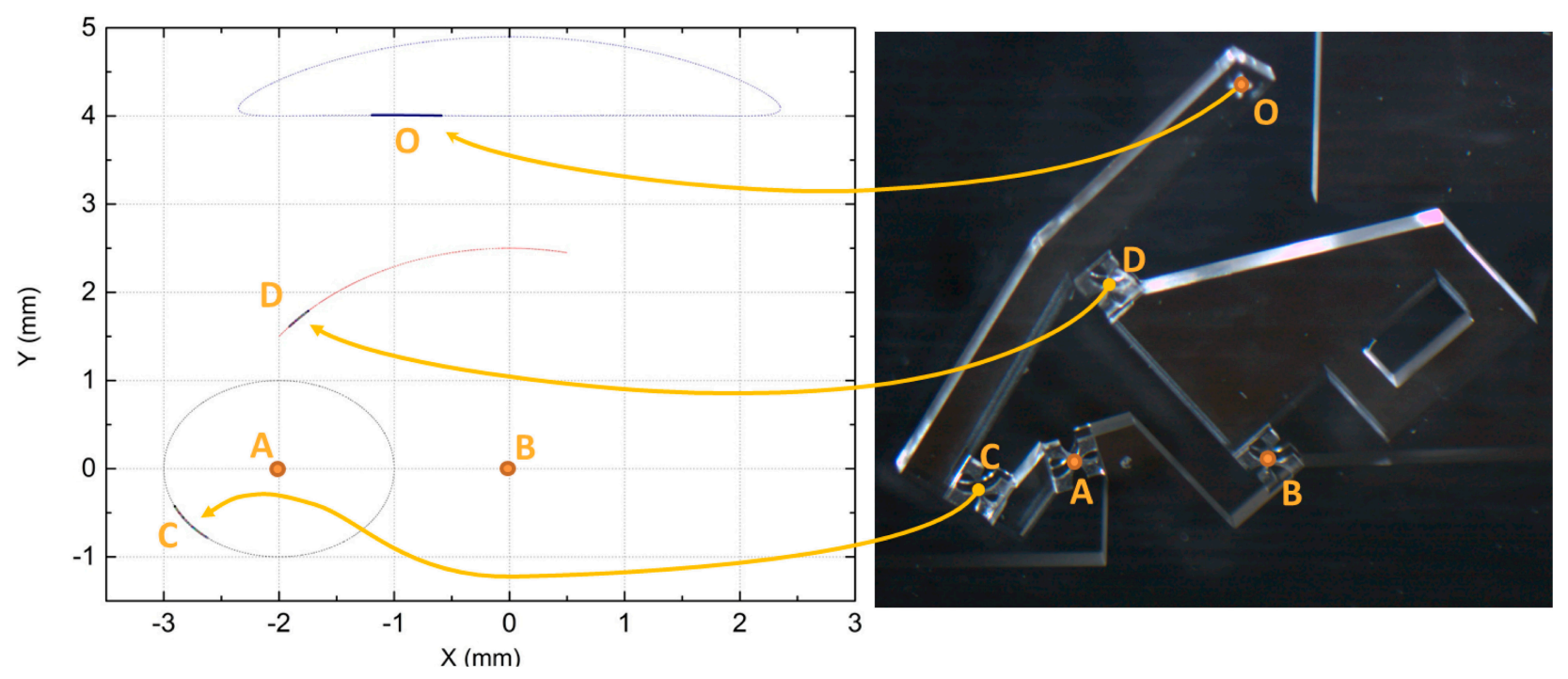

\section{Conclusions}

Among the various designs of flexure pivots, we have shown that the cross-spring pivot, in comparison to other elemental flexures used in microsystems design has superior performances in terms of stiffness contrast, range of motion and approximation of an ideal one-degree of freedom pivot. To date, the manufacture of cross-pivot hinges has been limited by the lack of available, micro-manufacturing processes, suitable for producing three-dimensional structures.

Here, we have demonstrated that, using of femtosecond laser processing combined with chemical etching, such complex three-dimensional flexure pivots can be manufactured, offering new opportunities for novel monolithic flexure-based mechanisms, which were previously impossible to fabricate using conventional means. As an illustration of the potential of the femtosecond laser machining of glass substrates, we have shown the integration of this cross-spring pivot in a glass transparent Hoecken guidance mechanism.

To the best of our knowledge, this is the first example of monolithic and non-trivial three-dimensional hinges fabricated at the micro-scale.

\section{Acknowledgments}

The authors acknowledge the partial financial support of the European Commission (Femtoprint project, FP7-NMP, Factory of the Future initiative) and of the European Research Council (Galatea project, ERC-2012-StG-307442).

\section{Author Contributions}

Viktor Tielen, during his master thesis, performed the experiments and fabricated the various flexures presented here. He optimized the design of the flexure to improve its stiffness characteristics 
and made the design of the Hoecken glass linkage. Yves Bellouard designed and supervised the research, wrote the manuscript and performed the comparative theoretical analysis between flexures.

\section{Conflicts of Interest}

The authors declare no conflict of interest.

\section{References and Notes}

1. Smith, S.T. Flexures: Elements of Elastic Mechanisms; CRC Press: Boca Raton, FL, USA, 2000.

2. Lobontiu, N. Compliant Mechanisms: Design of Flexure Hinges; CRC Press: Boca Raton, FL, USA, 2010.

3. Howell, L.L. Compliant Mechanisms; John Wiley \& Sons: Hoboken, NJ, USA, 2001.

4. Sears, J.E. A symmetrically opening optical slit. J. Sci. Instrum. 1933, 10, 376-377.

5. Haringx, J.A. The cross-spring pivot as a constructional element. Appl. Sci. Res. 1949, 1, 313-332.

6. Wittrick, W.H. The theory of symmetrical crossed flexure pivots. Aust. J. Sci. Res. Ser. A Phys. Sci. 1948, 1, 121-134.

7. Mesnager, A. Sur les articulations à lames flexibles. Comptes rendus Hebdomadaires des Séances de l'Académie des Sciences 1903, 137, 908-909. (In French)

8. Jones, R. V. An optical slit mechanism. J. Sci. Instrum. 1952, 29, 345-350.

9. Schellekens, P.; Rosielle, N.; Vermeulen, H.; Vermeulen, M.; Wetzels, S.; Pril, W. Design for precision: Current status and trends. CIRP Ann. Manuf. Technol. 1998, 47, 557-586.

10. Breguet, J.-M.; Henein, S.; Kjelberg, I.; Gumy, M.; Glettig, W.; Lecomte, S.; Boiko, D.; Mitev, V. Tunable extended-cavity diode laser based on a novel flexure-mechanism. Int. J. Optomechatronics 2013, 7, 181-192.

11. Marcinkevičius, A.; Juodkazis, S.; Watanabe, M.; Miwa, M.; Matsuo, S.; Misawa, H.; Nishii, J. Femtosecond laser-assisted three-dimensional microfabrication in silica. Opt. Lett. 2001, 26, 277-279.

12. Kiyama, S.; Matsuo, S.; Hashimoto, S.; Morihira, Y. Examination of etching agent and etching mechanism on femotosecond laser microfabrication of channels inside vitreous silica substrates. J. Phys. Chem. C 2009, 113, 11560-11566.

13. Bellouard, Y.; Said, A.; Dugan, M.; Bado, P. Monolithic Three-Dimensional Integration of Micro-Fluidic Channels and Optical Waveguides in Fused Silica. In Proceedings of Materials Research Society Symposium, Warrendale, PA, USA, 2003; pp. 63-68.

14. Bellouard, Y.; Champion, A.; Lenssen, B.; Matteucci, M.; Schaap, A.; Beresna, M.; Corbari, C.; Gecevicius, M.; Kazansky, P.; Chappuis, O.; Kral, M.; Clavel, R.; Barrot, F.; Breguet, J.-M.; Mabillard, Y.; Bottinelli, S.; Hopper, M.; Hoenninger, C.; Mottay, E.; Lopez, J. The Femtoprint Project. J. Laser Micro/Nanoeng. 2012, 7, 1-10.

15. Bellouard, Y.; Said, A.; Bado, P. Integrating optics and micro-mechanics in a single substrate: A step toward monolithic integration in fused silica. Opt. Express 2005, 13, 6635-6644.

16. Lenssen, B.; Bellouard, Y. Optically transparent glass micro-actuator fabricated by femtosecond laser exposure and chemical etching. Appl. Phys. Lett. 2012, 101, 103503. 
17. Cheng, Y.; Sugioka, K.; Midorikawa, K.; Masuda, M.; Toyoda, K.; Kawachi, M.; Shihoyama, K. Three-dimensional micro-optical components embedded in photosensitive glass by a femtosecond laser. Opt. Lett. 2003, 28, 1144-1146.

18. Juodkazis, S.; Yamasaki, K.; Mizeikis, V.; Matsuo, S.; Misawa, H. Formation of embedded patterns in glasses using femtosecond irradiation. Appl. Phys. A. 2004, 79, 1549-1553.

19. Sugioka, K.; Masuda, M.; Hongo, T.; Cheng, Y.; Shihoyama, K.; Midorikawa, K. Three-dimensional microfluidic structure embedded in photostructurable glass by femtosecond laser for lab-on-chip applications. Appl. Phys. A: Mater. Sci. Process. 2004, 79, 815-817.

20. Hasegawa, S.; Hayasaki, Y. Holographic vector wave femtosecond laser processing. Int. J. Optomechatronics 2014, 8, 73-88.

21. Bellouard, Y. On the bending strength of fused silica flexures fabricated by ultrafast lasers. Opt. Mater. Express 2011, 1, 816-831.

22. While it seems that the paternity of this linkage is to be attributed to Karl Hoecken (see [24]), the name Hoecken seems often misspelled and sometimes, this linkage is referred as Hoeken or Hoekens linkage, see for instance [23].

23. Lu, S.; Zlatanov, D.; Ding, X.; Molfino, R. A new family of deployable mechanisms based on the Hoekens linkage. Mech. Mach. Theory 2014, 73, 130-153.

24. Kerle, H. About Karl Hoecken and Some of His Works on Mechanisms. In Explorations in the History of Machines and Mechanisms: Proceedings of HMM2012; Koetsier, T., Ceccarelli, M., Eds.; Springer Netherlands: Dordrecht, The Netherlands, 2012; Volume 15, pp. 123-134.

25. Loncaric, J. Normal forms of stiffness and compliance matrices. IEEE J. Robot. Autom. 1987, 3, 567-572.

26. Ding, X.; Selig, J.M. On the compliance of coiled springs. Int. J. Mech. Sci. 2004, 46, 703-727.

27. Bellouard Y. Microrobotics: Methods and Applications; CRC Press: Boca Raton, FL, USA, 2010.

28. Von Mises R. Motorrechnung, ein neues hilfsmittel in der mechanik. J. Appl. Math. Mech./Z. Angew. Math. Mech. 1924, 4, 155-81. (In German)

29. Shusheng, B.; Hongzhe, Z.; Jingjun, Y. Modeling of a cartwheel flexural pivot. J. Mech. Des. 2009, 131, 061010.

30. Bellouard, Y.; Said, A.; Dugan, M.; Bado, P. Fabrication of high-aspect ratio, micro-fluidic channels and tunnels using femtosecond laser pulses and chemical etching. Opt. Express 2004, 12, 2120-2129.

(C) 2014 by the authors; licensee MDPI, Basel, Switzerland. This article is an open access article distributed under the terms and conditions of the Creative Commons Attribution license (http://creativecommons.org/licenses/by/3.0/). 